\title{
Joseph A. Bellanti (ed) Immunology IV: Clinical Applications in Health and Disease
}

\author{
Associate Editors Alejandro Escobar-Gutierrez and George C. Tsokos, \\ I Care Press Bethesda MD, 2012, 1,063 pages. \$99
}

\section{E. Richard Stiehm}

Received: 2 January 2012 / Accepted: 3 January 2012 /Published online: 23 February 2012

(C) Springer Science+Business Media, LLC 2012

This is an updated expanded revision of Dr. Bellanti's Immunology I, II, and III texts published in 1971, 1978, and 1985. The first edition was a single-column, 584-page book while this edition is double columned and has 1,063 pages. In the 27 years since these editions, clinical immunology has exploded with new illnesses, new diagnostic tests, new treatments, and improved prognoses. The editors, along with the help of 38 contributors, do an admirable job of updating the field for medical students, residents, fellows, and sophisticated clinicians.

As in the earlier volumes, there are three sections: the first is The Principles of Immunology (ten chapters) including basic immunology, developmental immunology, innate immunity, complement, inflammation, $\mathrm{T}$ cells and lymphocytes, B cells and immunoglobulins, mucosal immunity, cytokines and chemokines, and immunogenetics. Next is Mechanisms of Response (five chapters) including immunomodulation and responses to bacteria, virus, fungi, and parasites. The final section is Clinical Applications (ten chapters) including immunodeficiencies, hypersensitivity, autoimmunity, cancer, transplantation, laboratory diagnosis, and molecular biology. Appendices include tables of leukocyte antigens (CDs), interleukins, cytokines and chemokines, and acronyms.
Rarely does an editor devotes as much to a text as has Dr. Bellanti; he authored or coauthored 15 of the chapters and supervised and selected most of the clinical photos, illustrations, clinical cases, and summaries.

The text is lavishly illustrated: nearly every page has a photograph, a color figure, a short table, or a highlighted box for complex concepts. The emphasis as a teaching text is manifested by inserts or sections on case reports, clinical relevance, key points, study questions/critical thinking, and suggested readings (instead of detailed references). An online downloadable edition comes with purchase of the book.

The book is too detailed for an introductory text, too short for the advanced immunologist seeking cutting edge advances (with references), and too general for the clinician seeking diagnostic guidelines and evidence-based treatment options.

Who should buy this book? Immunology IV is ideal for an advanced course in immunology, for hospital and departmental libraries, for specialists and for physicians studying for board exams in allergy and immunology, rheumatology, or hematology. The online illustrations will be particularly useful to anyone preparing a presentation on any aspect of clinical immunology. And compared with competing texts, the price of $\$ 99$ is right. 